\title{
Determinação da demanda hídrica através do uso de tanques evaporímetros
}

\author{
Determination of hydric demand through the use of evaporimeters tanks \\ Determinación de la demanda hídrica mediante el uso de depósitos de evaporímetros
}

Recebido: 09/12/2021 | Revisado: 14/12/2021 | Aceito: 17/12/2021 | Publicado: 01/01/2022

\author{
Eletisanda das Neves \\ ORCID: https://orcid.org/0000-0002-4055-6658 \\ Universidade do Estado de Mato Grosso, Brasil \\ E-mail: eletisanda@unemat.br \\ Monica Isabelli de Almeida Gutierres \\ ORCID: https://orcid.org/0000-0003-2948-5358 \\ Universidade do Estado de Mato Grosso, Brasil \\ E-mail: monica.gutierres@unemat.br \\ Patrícia Ferreira da Silva \\ ORCID: https://orcid.org/0000-0002-4580-2336 \\ Universidade Federal de Campina Grande, Brasil \\ E-mail: patrycyafs@yahoo.com.br \\ Tulio Martinez Santos \\ ORCID: https://orcid.org/0000-0003-1103-9464 \\ Universidade do Estado de Mato Grosso, Brasil \\ E-mail: tulio.martinez@unemat.br
}

\begin{abstract}
Resumo
O manejo eficiente da água no atual cenário de escassez hídrica mundial, é uma constante, nesse sentido a busca de formas de manejo de sistema de irrigação que prezem a economia dos recursos hídricos é essencial. Assim a quantificação a demanda hídrica das culturas com o uso de tanques evaporímetros esta cada vez mais em evidencia, por ser um método indireto e de baixo custo, ainda mais em se tratando de tanques alternativos. Assim, objetivou-se com esse trabalho estudar a determinação da demanda hídrica através do uso de tanques evaporímetros que seja de baixo custo e possíveis de aplicação em qualquer propriedade agrícola visando o manejo racional da irrigação frente a problemática de escassez hídrica. $\mathrm{O}$ trabalho versa em um levantamento bibliográfico em banco de dados acadêmicos: Google acadêmico, banco de Teses e Dissertações (BDTD) e livros, scielo e Science Direct, com palavras chaves sobre o tema no período de 1997 a 2021. Os evaporímetros são eficientes na quantificação da necessidade hídrica em diferentes regiões e culturas. Evaporímetros alternativos são de baixo custo e podem ser utilizados pelos produtores de grande, médio e pequeno porte em suas propriedades. Existem diversos trabalhos sobre a quantificação da demanda hídrica em diferentes culturas com o uso evaporímetros tanto a nível nacional quanto internacional. $\mathrm{O}$ manejo da irrigação com uso de tanques evaporímetros pode ser de baixo custo e acessível. Atender a demanda hídrica das culturas é essencial para ter boas produtividades e evitar desperdícios dos recursos hídricos no atual cenário de escassez.
\end{abstract}

Palavras-chave: Manejo de irrigação; Tanque alternativo; Baixo custo; Evapotranspiração; Uso racional da água.

\begin{abstract}
The efficient management of water in the current scenario of global water scarcity is a constant, in this sense, the search for ways to manage an irrigation system that value the economy of water resources is essential. Thus, the quantification of the water demand of cultures using evaporimeter tanks is increasingly in evidence, as it is an indirect and low-cost method, especially when it comes to alternative tanks. Thus, the objective of this work was to study the determination of water demand through the use of low cost evaporimeter tanks that can be applied in any agricultural property aiming at the rational management of irrigation in face of the problem of water scarcity. The bibliographic survey work in an academic database: Academic Google, Theses and Dissertations and books, Science and Science Direct, with keywords on or subject not lost from 1997 to 2021. Evaporimeters are efficient in quantifying the water requirement in different regions and cultures. Alternative Evaporimeters are inexpensive and can be used by large, medium and small producers on their properties. There are several works on the quantification of water demand in different cultures with the use of evaporimeters both nationally and internationally. Irrigation management using evaporimeter tanks can be low cost and accessible. Meeting the water demand of crops is essential to have good yields and avoid wasting water resources in the current scenario of scarcity.
\end{abstract}

Keywords: Irrigation management; Alternative tank; Low cost; Evapotranspiration; Rational use of water. 


\begin{abstract}
Resumen
La gestión eficiente del agua en el escenario actual de escasez global de agua es una constante, en este sentido, la búsqueda de formas de gestionar un sistema de riego que valore la economía de los recursos hídricos es fundamental. Así, la cuantificación de la demanda de agua de cultivos mediante tanques evaporimétricos es cada vez más evidente, por tratarse de un método indirecto y de bajo coste, especialmente cuando se trata de tanques alternativos. Así, el objetivo de este trabajo fue estudiar la determinación de la demanda de agua mediante el uso de tanques evaporimétricos de bajo costo que se pueden aplicar en cualquier finca agrícola con el objetivo de la gestión racional del riego ante el problema de escasez de agua. El trabajo de relevamiento bibliográfico en una base de datos académica: Google académico, Tesis y disertaciones y libros, Science and Science Direct, con palabras clave sobre o tema no perdido desde 1997 hasta 2021. Los evaporadores son eficaces para cuantificar las necesidades de agua en diferentes regiones y culturas. Los evaporadores alternativos son económicos y pueden ser utilizados por grandes, medianos y pequeños productores en sus propiedades. Existen varios trabajos sobre la cuantificación de la demanda de agua en diferentes culturas con el uso de evaporímetros tanto a nivel nacional como internacional. La gestión del riego mediante tanques evaporimétricos puede ser de bajo costo y accesible. Satisfacer la demanda de agua de los cultivos es fundamental para tener buenos rendimientos y evitar el derroche de recursos hídricos en el actual escenario de escasez.
\end{abstract}

Palabras clave: Manejo de riego; Tanque alternativo; Bajo costo; Evapotranspiración; Uso racional del agua.

\title{
1. Introdução
}

Para um manejo racional dos recursos hídricos na agricultura irrigada, é fundamental a obtenção de dados meteorológicos para a cooperação no gerenciamento e eficiência de uso dos recursos hídricos. Em função disso, a utilização constante dos dados de evapotranspiração (ET) é essencial para as manejo de irrigação de elevada eficiência, estimativas de balanço hídrico e zoneamento climático ou mesmo agroclimático (Franco et al., 2015).

De acordo com Miranda et al. (2010) o manejo planejado de irrigação depende do conhecimento de parâmetros como a evaporação de água de solo, e lagos, assim como da evapotranspiração das plantas, esses dois processos ocorrem em decorrência da precipitação sobre a superfície retorna para a atmosfera. O manejo da irrigação exige a quantificação da quantidade de água indispensável para as plantas em seu planejamento, da qual a evapotranspiração, ou seja, somatório da evaporação mais a transpiração é um dos principais componentes.

Uma irrigação eficiente pode ser manejada por meio de diferentes métodos, dentre eles o manejo via, solo, via planta e o manejo via clima. O manejo via clima se utiliza de parâmetros como a evapotranspiração de referência, quantificada por métodos de campo, diretos, indiretos e por equações empíricas. Dentre os métodos considerados indiretos pode-se destacar o uso de tanques evaporimétricos que quantificam a evaporação e utilizando fatores de ajuste conforme a região, velocidade do vento, área coberta ou nua e distância da borda podem ser utilizadas para quantificar a evapotranspiração de referência de uma região, localidade ou mesmo propriedade. É um método considerado de baixo custo, pois mesmo tendo padrões quanto ao material de confecção dos tanques evaporimétricos e suas dimensões, diversas pesquisas tem sido desenvolvidas e constataram que pode-se construir esses tanques com materiais alternativos de baixo custo e fácil acesso para o produtor rural (oliveira et al., 2014; Seron et al., 2015; Labigalini et al., 2013; Sousa et al., 2016; Cunha, 2011; Gonçalves et al., 2014).

De acordo com Tagliaferre et al. (2012) a quantificação da demanda hídrica com uso do tanque classe A foi planejada para facilitar a obtenção dos valores de evapotranspiração de cultura (ETc), ou seja, multiplicando a evapotranspiração de referência obtida por diferentes formas pelo coeficiente de cultivo da cultura a ser irrigada naquela fase de desenvolvimento (Bernardo et al., 2019).

Dada a relevância dessa temática, objetivou-se com esse trabalho estudar a determinação da demanda hídrica através do uso de tanques evaporimétricos que seja de baixo custo e possíveis de aplicação em qualquer propriedade agrícola visando o manejo racional da irrigação frente a problemática de escassez hídrica. 


\section{Metodologia}

Este trabalho trata-se de uma pesquisa documental em termos metodológicos, segue um paradigma bibliográfico, uma vez que foi realizada uma análise da teoria de várias literaturas.

Para estetrabalh de pesquisa bibliografica, utilizou-se da metodologia descrita por Tupich et al. (2017). Nesse sentido, os dados para análise foram resultantes de pesquisas em periódicos científicos, leituras de artigos já publicados em plataformas académicas digitais, tais como: Scholar Google;Google acadêmico, Biblioteca Digital Brasileira de Teses e Dissertações (BDTD) e livros, Scielo e Science Direct. Destaca-se ainda que optou-se por trazer uma abordagem com trabalhos compreendidos entre 1997 a 2021.

Foram utilizadas as seguintes strings de busca: "irrigação" AND "manejo", "tanques" AND "evaporimétricos", evaporimétricos” AND “evapotranspiração”, necessidade” AND “hídricas”, tanques” AND “baixo custo”.

Os critérios para inclusão de estudos manejo de irrigação, foram:

I) trabalhos que tratam sobre manejo de irrigação em culturas por tanque classe A;

II) trabalhos que tratam de uso de tanques evaporímetros para determinar evapotranspiração; e ,

III) trabalhos que tratam sobre uso de taque classe A de baixo custo, custo de tanque classe A, tanque alternativo, calibração e tanque classe A, evapotranspiração por tanque classe A alternativo.

O fluxo de seleção de referências foi realizado conforme descrito por Lima et al. (2017), utilizando-se o software Mendeley.

\section{Levantamento Bibliométrico}

\subsection{Irrigação}

Nos últimos cinco anos, seja para atender às necessidades das atividades humanas ou às necessidades de serviços ambientais, a demanda por recursos hídricos aumentou de forma significativamente. Esse aumento se deve ao crescimento populacional e econômico, que se reflete nas mudanças sociais, principalmente na compreensão humana da importância da água no ecossistema, o que desperta o interesse da comunidade científica e da população de modo geral em adotar sistemas de gestão hídricas mais eficazes. Ressalta-se que dentre as formas de consumo dos recursos hídricos a agricultura irrigada responde por cerca de $85 \%$ do total de água captada, inferindo-se que a sustentabilidade dos campos irrigados depende da utilização de sistemas de irrigação mais eficientes para a aplicação da água nas lavouras (Schaible \& Aillery, 2017).

Para Bernardo et al. (2019) a eficiência de aplicação dos sistemas de irrigação é uma das variáveis que deve ser analisada, porém outras questões como culturas a serem irrigadas, sistema de cultivo, custo de implantação e operação do sistema também devem integrar a solução do problema.

A agricultura irrigação não deve ser vista como a grande vilã, mais para isso é necessário um manejo de irrigação eficiente associado a utilização de sistemas de irrigação eficientes. Segundo a ANA (2017) a um sistema de irrigação para ser considerado eficiente deve utilizar técnicas e equipamentos que visem atender a demanda hídrica das plantas, com o propósito de fornecer água de acordo com as necessidades das culturas, evitando excesso ou déficit hídrico, gerando uma alta produtividade.

Peragón et al. (2015) e Shamir et al. (2015) relatam que a irrigação possui grande importância mundial uma vez que contribui diretamente com a produção de alimentos, nesse sentido presume-se que o nível atual de $40 \%$ aumente para $45 \%$ em 2030, especialmente em regiões áridas e semiáridas onde tem-se á irregularidade na precipitação tanto no tempo quanto no espaço. 
Diante do cenário de escassez hídrica global é essencial a utilização de irrigação visando promover o aumento da produção de alimentos, uma vez que com base nos dados da Organização das Nações Unidas para Agricultura e Alimentação (FAO), estima-se que, atualmente, 815 milhões de pessoas sofram de desnutrição (Ciceri \& Allanore, 2019). A demanda por recursos está projetada para aumentar na medida em que a população cresça até 2050, o que requer aumentos de 55\% na disponibilidade de água, $60 \%$ de alimentos e $80 \%$ de energia (Daher et al., 2019).

Se a demanda por alimentos aumentar, aumenta também nessa mesma proporção a demanda pelos recursos hídricos, dessa forma é fundamental buscar alternativas visando a redução desse consumo, em especial, no contexto da agricultura irrigada, uma das alternativas para isso é utilização de sistemas mais eficientes e manejo adequado a cada tipo de cultura e região.

Quando se fala em sistemas eficientes precisa-se entender que existem três métodos de irrigação dentre os quais se destacam a irrigação por sulco com eficiência de aplicação variando de 50-65\%; a irrigação por aspersão que possui eficiência de 65 a 90\% e a irrigação por microirrigação com eficiência variando de 90 a 100\% (Bernardo et al., 2019). Cabe salientar que para que esses métodos de irrigação se subdividam em diversos sistemas e cada um a depender de seu dimensionamento e correto manejo apresentam eficiências diferentes, assim quanto melhor e mais tecnificado for o sistema maior será sua eficiência e consequentemente menores serão as perdas, aumentando sua eficiência.

\subsection{Evapotranspiração}

A evapotranspiração é definida como sendo o processo simultâneo de transferência de água para a atmosfera por meio da evaporação de água dos corpos hídricos, do solo e da vegetação úmida assim como da transpiração de água pelas plantas, pode-se dizer ainda que a evapotranspiração se constitui em um é um processo físico no qual acontece a perda de água do solo por meio da evaporação e transpiração natural da planta durante seus processos fisiológicos, sendo fundamental para preservação do ciclo hidrológico do planeta (Sentelhas \& Angelocci, 2009; Pereira et al., 2017; Matzenauer, 2018).

A evaporação consiste em um processo endotérmico, onde se transforma a energia externa em calor latente (L). Ocorrendo em duas partes, a evaporação de água do solo acontece de acordo com a profundidade do solo e propriedade física do solo como tipo de solo, textura e entre outros, quando o solo está úmido, esses dois fatores determinam a duração da evaporação deste solo. Após isso, o solo já com sua superfície seca, a evaporação acontece através de difusão molecular da água por meio de condutividade hídrica, diminuindo assim a taxa de evaporação de forma considerável (Matzenauer, 2018).

A transpiração consiste um processo de evaporação da água que foi usada pela planta em seus processos metabólicos, perdendo água em diversas partes da planta durante esse processo, sendo que a folha é onde mais ocorre essa perda. Ocorre um aumento de transpiração da planta durante seu período de floração, porém a transpiração acontece todo o desenvolvimento da planta, sendo assim um dos componentes mais importante para as plantas (Santana, 2011).

São diversos os tipos de evapotranspiração sendo eles: a evapotranspiração potencial (ETP) ou evapotranspiração de referencia $\left(E_{0}\right)$, a evapotranspiração real $\left(E_{R}\right)$, a evapotranspiração de Oasis $\left(E_{0}\right)$ e a evapotranspiração da cultura $\left(E_{C}\right)$.

A evapotranspiração potencial (ET $\mathrm{P}_{\mathrm{P}}$ consiste basicamente na evapotranspiração que ocorre em uma vasta vegetação, que possui um crescimento ativo em área com cobertura vegetal do solo, não tendo nenhuma ou pouca deficiência de água, ou seja, em uma condição ótima, sendo que apenas o balanço vertical energético pode interferir nesse processo de evapotranspiração (Thornthwaite, 1948). A evapotranspiração potencial em condições ditas homogenias, , ou seja, área com extensa superfície natural, totalmente coberta por vegetação baixa, com altura uniforme e com elevado índice de área foliar (IAF), de crescimento ativo na fase adulta, sendo como espécies adotada a grama e a alfafa e solo com umidade próxima a capacidade de campo (Carvalho et al., 2011). 
A evapotranspiração de referencia $\left(\mathrm{ET}_{0}\right)$ consiste na transferência hídrica do sistema solo-planta para a atmosfera em condições ideais da superfície, que possui uma coberta vegetal com alta uniformidade e maior presença de folhas, podendo ter encoberto totalmente a superfície do solo e onde existe uma quantidade hídrica próxima a capacidade de campo do solo. Essa evapotranspiração é utilizada para estimar a necessidade hídrica e para o planejamento de sistema de irrigação e manejo da água (Hargreaves, 1994; Grismer, 2002).

Para Carvalho et al. (2011) a evapotranspiração de referência $\left(\mathrm{ET}_{0}\right)$, originalmente era conhecida sob o termo evapotranspiração potencial (ETp), é considerado um parâmetro agrometeorológico de grande relevância, especialmente, para planejamento e manejo dos sistemas de irrigação, assim, como, também, é considerada elemento climático de demanda hídrica, em função disso, sua aplicação em estudos meteorológicos, climatológicos e hidrológicos para as mais variadas regiões.

Segundo Souza et al. (2010), a $\mathrm{ET}_{0}$ consiste em um parâmetro essencial para o planejamento da irrigação em qualquer cultura e região, uma vez que é uma variável de fácil obtenção sendo influenciada apenas por fatores relacionados ao clima. Contudo, apesar de existirem diversos métodos de estimativa o método considerado mais prático é utilizando o tanque classe A.

A evapotranspiração de oásis $\left(\mathrm{ET}_{\mathrm{O}}\right)$ acontece em regiões onde o clima é muito quente, a pequena área irrigada que está em volta de uma enorme área seca, a evapotranspiração é dirigida por balanço vertical e horizontal de energia, acontecendo que a área tampão é incapaz de erradicar o calor excessivo da região mais quente (Pereira, 1997). Podemos dizer que a evapotranspiração de Oasis é aquela de uma pequena área úmida circundada por uma área extensa seca, de onde a energia de advecção, ou seja, calor sensível se origina, essa que aumenta a quantidade de energia disponível para a evapotranspiração potencial (Bernardo et al., 2019; Pereira, 1997).

A evapotranspiração da cultura $\left(\mathrm{ET}_{\mathrm{C}}\right)$ é também conhecida como "evapotranspiração potencial da cultura", consiste no estado ótimos de umidade e nutrição do solo de forma que apresenta a produção excelente da cultura no solo (Bernardo et al., 2019). Esse tipo de evapotranspiração é de extrema importância para os projetos de irrigação e manejo, sendo que por meio da evapotranspiração de referência junto com o coeficiente de ajuste da cultura, descobre-se a evapotranspiração da cultura. $\mathrm{O}$ resultado desse cálculo é utilizado para fornecer água necessária para o desenvolvimento da cultura (Carvalho et al., 2011).

A evapotranspiração de forma geral é a base para o manejo da irrigação Via Clima, dessa foram vários estudos ao longo desses anos vem sendo desenvolvidos, buscando métodos diferentes para se estimar ou mesmo quantificar essa variável. Existem diferentes metodologias para quantificar a evapotranspiração de referencia, sendo eles divididos em métodos diretos e métodos indiretos, além do uso de equações empíricas. De acordo com Allen et al. (1998) uma forma comumente utilizada para estimar a ETc, consiste basicamente em multiplicar a evapotranspiração de referência $\left(\mathrm{ET}_{0}\right)$, pelo coeficientes de cultura (Kc) para determinada fase de desenvolvimento. O coeficiente de cultura nada mais é do que a integração de varias características da cultura tais como: altura, albedo, propriedades aerodinâmicas da folha e evaporação do solo (Bernardo et al., 2019; Pereira, 1997; Esteves et al., 2010; santos et al., 2017).

A utilização da evapotranspiração seja de referencia ou da cultura são essências para o manejo da irrigação, uma vez que elevam em consideração fatores como evaporação, transpiração e as fases de desenvolvimento das culturas.

\subsection{Tanques evaporimetricos}

Os tanques evaporimetricos são utilizados para determinar a evaporação de água das superfícies e indiretamente contribuem para a quantificação da evapotranspiração de referencia. De acordo com Trenberth et al. (2011) a evaporação da água em suas condições naturais se configura um dos principais componentes do ciclo hidrológico, representando aproximadamente $63 \%$ das chuvas, visto que as superfícies hídricas contribuem anualmente para o retorno de agua para atmosfera. 
Esses tanques, mais especificamente, o tanque classe A, foi desenvolvidos pelo Serviço Meteorológico NorteAmericano (U.S.W.B.) com a finalidade de auxiliar na estimativa da evapotranspiração de referencia de uma dada região, sendo este equipamento utilizado em larga abrangência em todo o mundo, incluído o Brasil (Pereira et al., 2015).

Sousa et al., (2016) relatam que a estimativa da $\mathrm{ET}_{0}$ com o uso do tanque Classe A (TCA) é baseado no princípio de que a água presente em seu interior não deve oferecer nenhuma dificuldade ao processo evaporativo. Assim, a evaporação obtida com o uso desse equipamento será maior quando comparada à perda efetiva de uma cultura, mesmo estando ela em condições satisfatórias, ou seja, ótimas de suprimento de hídrico. Baseado nisso, a quantificação da evapotranspiração de referencia $\left(\mathrm{ET}_{0}\right)$ utilizando o tanque classe $\mathrm{A}$, ou como é conhecido evaporímetros, depende do coeficiente de correção do tanque (Kp) que é função da velocidade do vento, da umidade relativa do ar e do tamanho da bordadura que o circunda.

Existes diferentes modelos de tanques evaporimétricos, no entanto, no Brasil o de maior utilização tem sido o tanque classe A de formato cilíndrico, com diâmetro de $121 \mathrm{~mm}$ e $25,5 \mathrm{~cm}$ de profundidade construído de com material galvanizado, calibre 22, e montado sobre plataforma de madeira com $15 \mathrm{~cm}$ de altura. Cabe destacar que esse tanque deve ser preenchido com água até $5 \mathrm{~cm}$ da borda e o nível da água não deve ficar abaixo de $7,5 \mathrm{~cm}$. Necessitando de renovação de forma regular da água (Pereira et al. 1997).

$\mathrm{O}$ tanque classe A, tem sido utilizado em projetos de irrigação devido ao seu custo reduzido e uma fácil manipulação. Havendo um grande benefício de medir a evaporação de uma superfície de água livre, alistada com os efeitos agregados a radiação solar, velocidade do vento, temperatura e umidade relativa do ar (Bernardo et al., 2019).

\subsection{Necessidade da elaboração de tanques para diminuir o custo}

A utilização de tanques evaporimétricos à preços mais acessíveis, apresentam várias vantagens quando comparados a tanques evaporimétricos de aço galvanizado para determinar a evaporação. Normalmente esses equipamentos são utilizados por empresas de pesquisa ou instituições privadas que investem altos valores no monitoramento do contínuo solo-água-plantaatmosfera (Valeriano et al., 2018).

De acordo com Coelho et al. (2017) quando a produção é pequena escala, a elaboração de tanques a baixo custo, pode se tornar uma alternativa técnica de qualidade, pois muitas vezes, os produtores carecem de informações locais para o dimensionamento e manejo da irrigação como um todo, assim como de poder aquisitivo para adquirir equipamentos, visto que, pequenos e médios produtores, por muitas vezes, se encaixam como produtores da agricultura familiar. Cabe salientar ainda que mesmo agricultores de grande porte com poder aquisitivo sempre buscam forma de baratear seus custos, e a utilização de equipamento alternativos que oferecem os mesmos resultados é uma constante.

O desenvolvimento de pesquisas de inovação tecnológica destinada à novos projetos na área da irrigação, é de suma importância visto dada a maior vulnerabilidade ao clima nos últimos anos, principalmente à irregularidade de distribuição das chuvas (Gurski et al., 2018). Desse modo, estudos que avaliam a precisão e a eficácia de métodos alternativos da aferição da evapotranspiração e/ou auxiliam o manejo da irrigação, podem proporcionar aos produtores a adotarem critérios técnicos e coesos no manejo da irrigação (Araújo et al., 2019; Peixoto et al., 2016).

Lisboa et al. (2011) afirmam em seus estudo sobre tanque evaporímetros alternativos e equações para estimativa da evapotranspiração de referencia na região norte de $\mathrm{MG}$, que a utilização de tanques alternativos visando quantificar a evaporação e indiretamente estimar a evapotranspiração de referencia $\left(\mathrm{ET}_{0}\right)$ pode ser uma ferramenta útil para o manejo da irrigação, especialmente, para pequenos agricultores que não têm acesso aos dados meteorológicos necessários para o uso das equações empíricas. Os tanques alternativos apresentam a vantagem da medição direta da evaporação, que se correlaciona com a evapotranspiração das culturas. 
Quando se analisa a eficiência da utilização desses tanques alternativos com equações empíricas e o tanque classe A padrão, observa-se boa correlação entre os dados de evapotranspiração estimada, evidenciando que esses equipamento podem ser utilizados pelos produtores para auxiliar no manejo da irrigação, sem, no entanto, causar problemas as culturas quanto ao fornecimento hídrico (Lisboa et al., 2011; Oliveira et al., 2011; Tagliaferre et al., 2011; Seron et al., 2015; Bandeira et al., 2011; Coelho et al., 2014).

Os tanques evaporímetros alternativos possui diversas denominações tais como: minievaporímetro; minitanque; tanque classe A alternativo, tanque reduzido dentre outros e são confeccionados com os mais diversos tipos de materiais como alumínio; PVC; amianto; e mesmo aço galvanizado. O valor do tanque classe A padrão é de aproximadamente R $\$ 8400,00$, o que limita o uso por pequenos produtores, no entanto, diversos estudos com vários pesquisadores envolvidos têm trabalhado com a finalidade de desenvolver evaporímetros de baixo custo em média ( $\mathrm{R} \$ 500,00)$, de fácil manuseio e boa precisão na medição da evaporação de uma superfície livre de água, facilitando o acesso e sua difusão junto aos produtores (Seron et al., 2015; Oliveira et al., 2011; Tagliaferre et al., 2011; Santos et al., 2004; Bandeira et al., 2011;).

Esses tanques de baixo custo tem sido testado para quantificação de evapotranspiração de referencia e utilizados para manejo de irrigação nas mais variadas culturas, desde culturas perenes ou mesmo anuais, oferecendo resultados satisfatórios para o suprimento da demanda hídrica das culturas, nas mais diferentes fases fenológicas (Bandeira et al., 2011; Santos et al., 2004; Gonçalves et al., 2014; Pereira et al., 2017; Lisboa et al., 2011)

\subsection{Necessidade hídrica das culturas}

A necessidade hídrica das culturas consiste em se quantificar a quantidade de água que ela necessita em um determinado período/fase fenológica ou mesmo ao longo de todo o seu ciclo produtivo, para que não ocorra a delimitação de seu desenvolvimento e produção, porém nas condições climáticas ideais ela é o fornecimento de água necessário para atender a demanda de evapotranspiração das culturas (Carvalho et al., 2013).

O conhecimento da necessidade hídrica é fundamental para implantar uma cultura no campo, o produtor necessita de conhecimento para estimar o volume de água a ser aplicado ao longo de seu ciclo de desenvolvimento. A determinação de períodos em que ocorrera a escassez de água é de suma importância, pois o produtor deve definir alternativas para amenizar as perdas do seu rendimento (Carvalho et al., 2013; Silva et al., 2015).

Neumaier et al. (2020) afirmam que a exemplo tem-se a cultura da soja que evidencia elevada necessidade hídrica variando de aproximadamente 450 a $800 \mathrm{~mm}$ de água durante todo o seu ciclo, para atingir seu pleno desenvolvimento e alto potencial produtivo. Sendo seu período crítico as fases de emergência, floração e enchimento dos grãos (Silva et al., 2015). Em função das janelas de produção nas diversas regiões produtoras ocorrer durante o período chuvoso, entretanto, acontecesse veranicos que são períodos sem chuvas, conforme a prorrogação desse período pode-se acarretar severos prejuízos nas lavouras (Silva et al., 2015).

Para a cultura do milho tem-se também grande demanda hídrica, necessitando de 250 a $500 \mathrm{~mm}$ de água durante todo o seu ciclo, sendo sensível ao estresse hídrico causado pela baixa disponibilidade de água no solo, especialmente ao decorrer do seu período crítico, que ocorre do início da floração ao final do enchimento do grão (Hernández et al., 2015). A ocorrência de estresse hídrico nesse período para a cultura causa diversos prejuízos, no desenvolvimento da planta gerando como consequência a perda da produtividade, a redução da altura da planta e do diâmetro do caule (Guimarães; Rocha; Paternian, 2019).

Quando pensa em culturas como o algodoeiro Carvalho et al. (2013) e Zonta et al. (2017) afirmam que a demanda hídrica varia de 700 a $1.300 \mathrm{~mm}$ de água ao decorre do seu ciclo. Aproximadamente $80 \%$ da estrutura reprodutiva surgem entre 60 a 80 dias após sua emergência necessitando assim da alta disponibilidade de água. Nesse sentido, se durante suas 
diferentes fases fenológicas ocorrerem deficiência hídrica tem-se perdas severas em seu desenvolvimento e produção (SILVA et al., 2015).

A necessidade hídrica do girassol é de aproximadamente 600 a $1.000 \mathrm{~mm}$ de água ao longo do seu ciclo, a evapotranspiração nos períodos de florescimento aumenta podendo atingir em torno de 12 e $15 \mathrm{~mm}$.dia ${ }^{-1}$ (Doorenbos \& Kassam, 1979). A limitação da disponibilidade de água nesse período pode afetar o desenvolvimento da planta acarretando perdas na produtividade (Gazzola et al., 2012).

A cultura do feijão possui uma alta exigência por climas tropicais com temperaturas em média de $25^{\circ} \mathrm{C}\left(18^{\circ}\right.$ a $\left.30^{\circ}\right)$, sua necessidade hídrica ao longo do ciclo de $741,11 \mathrm{~mm}$ distribuída na cultura, sendo uma planta muito sensível ao estresse hídrico seu período crítico ocorre durante a emergência e a floração (Simeão et al., 2013). Os danos através do estresse hídrico são verificados na floração quando surge a queda de flores reduzindo a produtividade da planta (Simeão et al., 2013).

A cultura do amendoim necessita em média de $500 \mathrm{~mm}$ de água ao decorrer do seu ciclo (Coelho et al., 2017). O déficit hídrico causa grandes perdas na produtividade do amendoim em todo o mundo. Em regiões onde ocorre veranicos longos é de grande importância a utilização da irrigação, para reverter a situação da falta de água da cultura (Pereira et al., 2016).

O déficit hídrico é a consequência do balanço hídrico, em que a quantidade de água que entra no sistema através da precipitação é menor do que a quantidade de água perdida pelas plantas por evaporação e transpiração (Duarte et al., 2012).

\section{Conclusão}

Os evaporímetros são eficientes na quantificação da necessidade hídrica em diferentes regiões e culturas.

Evaporímetros alternativos são de baixo custo e podem ser utilizados pelos produtores de grande, médio e pequeno porte em suas propriedades.

Existem diversos trabalhos sobre a quantificação da demanda hídrica em diferentes culturas com o uso evaporímetros tanto a nível nacional quanto internacional.

O manejo da irrigação com uso de tanques evaporímetros pode ser de baixo custo e acessível.

Atender a demanda hídrica das culturas é essencial para ter boas produtividades e evitar desperdícios dos recursos hídricos no atual cenário de escassez.

Recomendar-se maior difusão desses tanques alternativos de baixo custo junto aos produtores, visto que esses são os maiores interessados.

Recomenda-se mais estudos sobre a temática para reforçar com mais bases de dados e palavras chaves.

\section{Agradecimentos}

A Universidade do Estado do Mato Grosso.

\section{Referências}

Allen, R. G., Pereira, L. S., Raes, D. \& Smith, M. (1998) Crop evapotranspiration: Guidelines for computing crop water requirements. FAO, 300 p. (FAO Irrigation and Drainage Paper, 56).

ANA - Agência Nacional de Águas. (2017) Atlas irrigação: uso da água na agricultura irrigada. Brasília,

Araujo, A. L. G., Rubio Neto, A., Almeida, A. M., Guimaraes, J. J., Cantuario, F. S., Salomão, L. C., Curvelo, C. R. S., Luz, J. M. Q., Pereira, A. I. A., Souza, M. L. C. \& Santos, J. C. C. (2019) Construção de sistema de irrigação para experimentos em parcelas subdivididas. Informe Goiano Circular de Pesquisa Aplicada, 07(10)01-06.

Bandeira, G. R. L., Pinto, H. C. S., Magalhães, P. S., Aragão, C. A., Queiroz, S. O. P., Souza, E. R. \& Seido, S. L. (2011). Manejo de irrigação para cultivo de alface em ambiente protegido. Horticultura Brasileira 29(1) 237-241. 
Bernardo, S., Soares, A. A., Mantovani, E. C. \& Silva, D. D. (2019). Manual de irrigação. UFV, 545p.

Carvalho, I. R., Korcelski, C., Pelissari, G., Hanus, A. D. \& Rosa, G. M. (2013). Demanda hídrica das culturas de interesse agronômico. Enciclopédia biosfera. 9(17) $969-985$.

Carvalho, L. G. C., Rios, G. F. A., Miranda, W. L. \& Castro Neto, P. (2011). Evapotranspiração de Referência: Uma Abordagem Atual de Diferentes Métodos de Estimativa. Pesquisa Agropecuária Tropical. 41(3)456-465.

Ciceri, D. \& Allanore, A. (2019). Local fertilizers to achieve food self-sufficiency in Africa. Science of The Total Environment, 648(1)669-680.

Coelho, A. P, Faria, R. T. \& Dalri, A. B. (2017) Ecophysiology and irrigation of the peanuts cultivated in second season. Applied Research \& Agrotechnology. 10(2) 119-126.

Coelho, R. F., Silva, A. J. P., Parizotto, I. \& Silva, T. S. M. (2014). Sistemas e manejo de irrigação de baixo custo para agricultura familiar. Cruz das Almas, BA: Embrapa Mandioca e Fruticultura.

Cunha, A. R. (2011). Coeficiente do tanque Classe A obtido por diferentes métodos em ambiente protegido e no campo. Semina: Ciências Agrárias. 32(2) 451-464.

Daher, B., Hannibal, B., Portney, K. E. \& Mohtar, R. H. (2019). Toward creating an environment of cooperation between water, energy, and food stakeholders in San Antonio. Science of The Total Environment, 651(2) 2913-2926.

Doorenbos, J. \& Kassam, A. H. (1979). Yield response to water. Roma: Food and Agriculture Organization of United Nations (FAO), Cap. Sunflower. 150152 .

Duarte, J. M. De L, Lima, A. D, Nascimento, R. S, Viana, T. V. De A, Saraiva, K. R. \& Azevedo, B. M. De. (2012). Eficiência do uso da água na produção de óleo do girassol (helliantusannuusL.), sob suspensão hídrica. Revista Brasileira de Agricultura Irrigada0.6(3)166 - 175.

Esteves, B.S., Mendonça, J.C., Sousa, E.F.\& Bernardo, S. (2010). Avaliação do Kt para estimativa da evapotranspiração de referência (ET ${ }_{0}$ ) em Campos dos Goytacazes, RJ. Revista Brasileira de Engenharia Agrícola e Ambiental. 14(3) 274-278.

Franco, R. A. M., Hernandez, F. B. T. \& Teixeira, A. H. C. (2015). Aplicação do algoritmo SAFER na determinação da evapotranspiração em condições de sazonalidade climática no noroeste paulista. Anais XVII Simpósio Brasileiro de Sensoriamento Remoto - SBSR, João Pessoa-PB, Brasil.

Gazzola, A., Ferreira, C. T. G., Cunha, D. A. \& Bortolini, E. (2012). A cultura do girassol. Universidade de São Paulo Escola Superior de Agricultura. Departamento de Produção Vegetal. Piracicaba, SP.

Gonçalves, F. V., Medici, L. O., Almeida, W. S., Carvalho, D. F., Santos, H. T. \& Gomes, D. P. (2014). Irrigação no cultivo orgânico de alface utilizando Irrigás, tanque Classe A e um sistema automático de baixo custo. Ciência Rural. 44(11) 1950-1955.

Grismer, M. E. et al. (2002). Pan evaporation to reference evapotranspiration conversion methods. Journal of irrigation and Drainage Engineering. 128(3), 180-184,

Guimarães, P. S., Rocha, D. S. \& Paterniani, M. E. A. G. Z. (2019). Conteúdo de carboidrato foliar em híbridos de milho submetidos à restrição hídrica. Evidencia. 19(2)93-112.

Gurski, B. C., Jerszurki, D. \& Souza, J. L. M. (2018). Alternative reference evapotranspiration methods for the main climate types of the state of Paraná, Brazil. Pesquisa Agropecuária Brasileira, 53(3) 1003-1010.

Hargreaves, G. H. (1994). Definig and usining reference evaporatranspiration. Journal of Irrigation and Drainage Engineering. 120(6) 1132-1139.

Hernández, M., Echarte, L., Della Maggiora, A., Cambareri, M., Barbieri, P. \& Cerrudo, D. (2015). Maize water use efficiency and evapotranspiration response to N supply under contrasting soil water availability. Field Crops Research.178(1)8-15.

Labigalini, I., Veiga, J. C., Pino, M. A. I. T.\&Lima, C. M. (2013). Construção e análise da eficiência de um mini-tanque evaporimétrico de baixo custo no manejo da irrigação. $5^{a}$ Jornada Científica e Tecnológica e $2^{o}$ Simpósio de Pós-Graduação do IFSULDEMINAS. 1(5)1-6.

Lima, A. O. De L., Mochón, L. G. \& Tamayo, C. B. (2017). Identificación de indicadores de resultado en salud en atención primaria. Una revisión de revisiones sistemáticas. Revista de Calidad Asistencial. 32(5) 278-288.

Lisboa, T. M., Batista, C. H., Aquino, L. A., Silva, H. R. F., Melo, V. L. \& Santos Junior, V. C. (2011). Tanque evaporimétrico alternativo e equações para estimativa da evapotranspiração de referência na região norte de MG. Revista Brasileira de Agricultura Irrigada.5(1)54-62.

Matzenauer, R. (2018). Evapotranspiração da cultura do milho. II- Relações com a evaporação do tanque classe "A" com a evaporação de referencia e com a radiação solar global, em três épocas de semeadura. Revista Brasileira de Agrometeorologia. 6(1)15-21.

Miranda, R. A. C., Oliveira, M. V. S.\& Silva, D. F. da. (2010). Ciclo hidrográfico planetário: abordagens e conceitos. Geo UERJ. 1(21) 109-119.

Neumaier, N., Farias, J. R. B., Nepomuceno, A. L., Mertz-Henning, L. M., Foloni, J. S. S., Moraes, L. A. C. \&Goncalves, S. L. (2020). Ecofisiologia da soja In: Seixas, C. D. S., Neumaier, N., Balbinot Junior, A. A., Krzyzanowski, F. C.\& Leite, R. M. V. B. De C. (Ed.). Tecnologias de produção de soja. Londrina: Embrapa Soja, 33-54. (Embrapa Soja. Sistemas de Produção, 17).

Oliveira, E. C., Carvalho, J. D. A, Almeida, E. F. A, Rezende, F. C., Santos, B. G. Dos. \& Mimura, S. N. (2014). Evapotranspiração da roseira cultivada em ambiente protegido. Revista Brasileira de Engenharia Agrícola e Ambiental. 18(5) 314-321. 
Oliveira, G. M., Leitão, M. M. V. B. R., Bispo, R. C., Santos, I. M. S. \& Almeida, A. C. (2011). Comparação entre métodos de estimativa da evapotranspiração de referência na região Norte da Bahia. Revista Brasileira de Agricultura Irrigada. 4(2)104-109.

Peixoto, T. D .C, Leviaen, S. L. A, Bezerra, A. H. F, Silva, S. T. A. \& Sobrinho, J. E. (2016). Coeficiente do tanque classe A para a região de Mossoró, RN. Revista Brasileira de Agricultura Irrigada.10(2) 515 - 521.

Peragón, J. M., Pérez-Latorre, F. J. Delgado, A. (2015). A GIS-based quality assessment model for olive tree irrigation water in southern Spain. Agricultural Water Management. 148(1) 232-240.

Pereira, P. C., Jardim, A. M. R. F., Lima, L.R., Queiroz, M. G., Silva, T. G. G. \& Morais, J. E. F. (2017) Evapotranspiração do girassol pelo método do tanque classe "a” na região do semiárido brasileiro. Revista Brasileira de Agricultura Irrigada.11(6) 1894 - 1903.

Pereira, A. R., Villa Nova, N. A. \& Sediyama, G. C. (1997) Evapotranspiração. FEALQ, 183p.

Pereira, J. W. L, Albuquerque, M. B., Melo Filho, P. A, Nogueira, R. J. M. C, Lima, L. M. \& Santos, R. C. (2016). Assessment of drought tolerance of peanut cultivars based on physiological and yield traits in a semiarid environment. Agricultural Water Management, 166(1)70-76.

Pereira, L. S., Allen, R. G., Smith, M. \& Raes, D. (2015). Crop evapotranspiration estimation with FAO56: Past and future. Agricultural Water Management, 147(2) 4-20.

Santana, M. J., Pereira, U. C., Beirigo J. D. C., Souza, S. S., Campos, T. M. \& Vieira, T. A. (2011). Coeficiente de cultura para o Tomateiro Irrigado. Irriga. 6(1) $11-20$

Santos, F. J. S., Lima, R. N., Rodrigues, B, H, N., Crísótomo, L. A., Souza, F. \& Oliveira, J. J. G. (2004) Manejo da Irrigação da Melancia: Uso do Tanque Classe A. Circular técnica 20. Embrapa 1(1) 1-13.

Santos, R. A., Santos, E. P., Sales, R. A. \& Santos, R. L. (2017). Estimativa da evapotranspiração de referência para o município de feira de santana (BA). Revista Brasileira de Agricultura Irrigada-RBAI.11(4) 1617 - 1626.

Schaible, G. D. \& Aillery, M. P. (2017). Challenges for US Irrigated Agriculture in the Face of Emerging Demands and Climate Change. In: SCHAIBLE, G. D., AILLERY, M. P. Competition for Water Resources. Elsevier, Cap. 2. 44-79.

Sentelhas, P. C. \& Angelocci, L. R. (2009). Balanço hídrico climatológico normal e sequencial de cultura e para manejo da irrigação. Piracicaba, ESALQ/USP, 40p.

Seron, C. C., Souza, A. H. C., Lorenzoni, M. Z., Rezende, R., Maller, A. \& Hachmann, T. L. (2015). calibração de um tanque reduzido em ambiente protegido. Anais Eletrônico. IX EPCC - Encontro Internacional de Produção Científica UniCesumar. 1 (9).4-8.

Shamir, E., Megdal, S. B., Carrillo, C., Castro, C. L., Chang, H., Chief, K., Corkhill, F. E., Eden, S., Georgakakos, K. P., Nelson, K. M. \& Prietto, J. (2015). Climate change and water resources management in the Upper Santa Cruz River, Arizona. Journal of Hydrology. 521(2) 18-33.

Silva, A.F., Sediyama, T. \& Borém, A. (2015). Exigências edafoclimáticas. In: Sediyama, T., Silva, F. \& Borém, A. (Eds.). Soja: do plantio à colheita. Viçosa: UFV, 54-65.

Simeão, M., Oliveira, A. E. S., Santos, A. R. B., Mousinho, F. E. P. \& Ribeiro, A. A. (2013). Determinação da ETc e Kc para o feijão - fava (Phaseolus lunatus L.) na região de Teresina, Piauí. Revista Verde. 8(2) 291 - 296.

Sousa I. F. De, Silva V. De P. R. Da, Sabino F. G., Netto A. De O. A., Silva B. K. N. \& Azevedo P. V. De. (2010).Evapotranspiração de referência nos perímetros irrigados do estado de Sergipe. Revista Brasileira de Engenharia Agrícola e Ambiental. 14(5) 633-644.

Sousa, I, F., Faccioli, G. G. \& Aguiar Netto, A. O. (2016). Avaliação do coeficiente do tanque classe "a" na estimativa da evapotranspiração de referência no baixo São Francisco, SE. Irriga, Edição Especial.47-58.

Tagliaferre, C., Oliveira, R.A., Sediyama, G. C., Cecon, P. R., Materán, F. J. V. \& Martinez, M. A. (2011). Influência da presença da bordadura e dos níveis de água na evaporação obtida em minievaporímetros. Revista Ceres. 58(2)161-167.

Tupich, F. L. B. (2015). Metanálise do ganho de produtividade da soja com aplicações de fluazinam para o controle do mofo branco. 2015.42 p. Dissertação (Mestrado em Agronomia) - Universidade Estadual de Londrina, Londrina, PR.

Thornthwaite, C. W. (1948). An approach toward a rational classification of climate. Geographical Review, 38(1)55-94.

Trenberth, K. E., Fasullo, J. T \&, Mackaro, J. (2011). Transportes de umidade atmosférica do oceano para a terra e fluxos de energia global em reanálises. Journal of Climate. 24(1)4907-4924.

Valeriano, T. T., Santana, M. J., Jesus, M. V. \& Leite, L. S. (2018). Manejo de irrigação para a alface americana cultivada em ambiente protegido. Nativa: Pesquisas Agrárias e Ambientais. 6(2) 118-123.

Zonta, J. H., Brandão, Z. N., Rodrigues, J. I. Da. S. \& Sofiatti, V. (2017). Cotton response to water deficits at different growth stages. Revista Caatinga. 30(4) 980-990. 